S Sii ${ }^{1}$, P Aspinall ${ }^{2,3}$, S Borooah ${ }^{4,5}$ and B Dhillon ${ }^{5,6}$

\section{Exploring factors predicting changes in patients' expectations and psychosocial issues during the course of treatment with intravitreal injections for wet age-related macular degeneration}

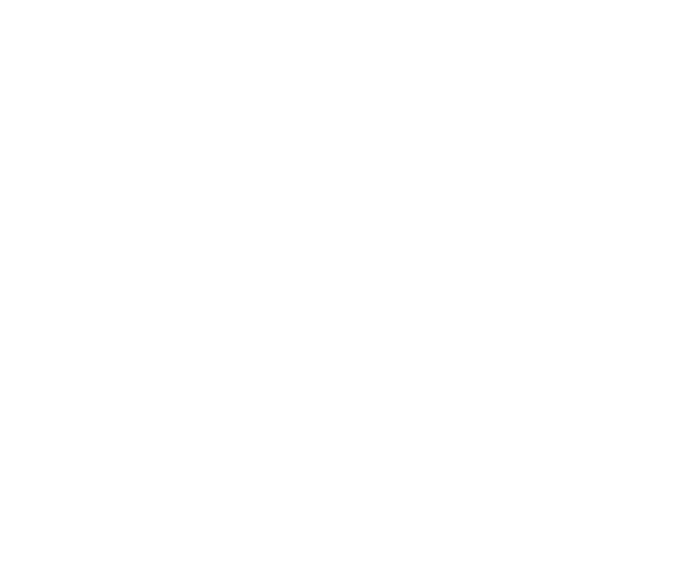

\begin{abstract}
Purpose Patients with wet age-related macular degeneration (AMD) often require long courses of treatment. We investigate the psychosocial issues that could hinder compliance, including patient expectations of treatment. The aims of this study were to explore the factors related to changes in patient expectations, pain, and anxiety during treatment.

Patients and methods A structured interview was carried out among 50 patients selected from the list attending the AMD unit at the Princess Alexandra Eye Pavilion (PAEP). The interview was based on a questionnaire. Additionally, a visual analogue scale was created as a tool for measuring patient expectations, pain, and anxiety. Data were analysed using multinomial regression analysis.

Results There were significantly more patients who had a fall in expectations $(P<0.05)$ during the course of treatment. A fall in expectations was found to be predicted by higher starting expectations $(P=0.00001)$, greater decline in visual acuity $(P=0.008)$, and perceived deterioration of vision after starting treatment $(P=0.013)$. Of the patients, $32 \%$ planned to stop attending for further injections. Planning to stop attending was correlated with worse final visual acuity $(P=0.026,95 \% \mathrm{CI})$. Pain and anxiety with intravitreal therapy (IVT) was significantly reduced when patients were accompanied to the clinic by a friend or relative $(P<0.01)$ using Pearson's correlation $(r=0.597)$.
\end{abstract}

Conclusion Patients require appropriate counselling at the start of a course of treatment to align expectations with perceived treatment outcomes in order to improve adherence. Additionally, a large minority of patients would consider stopping treatment. Patients' expectations should be assessed at relevant time points along a course of treatment. Eye (2018) 32, 673-678; doi:10.1038/eye.2017.271; published online 8 December 2017

\section{Introduction}

Age-related macular degeneration (AMD) is the commonest cause of blindness in the western world. ${ }^{1,2}$ In the United Kingdom, the burden of this disease is increasing and its prevalence is projected to continue to rise with an ageing population. ${ }^{3}$ Although wet AMD only accounts for $10 \%$ of all AMD cases, it is responsible for $90 \%$ of visual impairment caused by this disease. ${ }^{4}$ Anti-vascular endothelial growth factor (anti-VEGF) drugs such as ranibizumab and aflibercept have been shown to successfully prevent vision loss in the majority of patients with wet AMD. Treatment courses are expensive and often prolonged requiring repeated intraocular injections. ${ }^{5}$ The use of anti-VEGF drugs has increased by more than twofold from 2008 to 2015. ${ }^{3,4}$ A single ranibizumab injection costs the NHS $£ 742.17$ per patient. ${ }^{6}$ In view of cost of treatment and long trajectory of disease, it is therefore important to explore whether patient expectations of these injections are met. In addition, there are still significant gaps to bridge
${ }^{1}$ Department of Ophthalmology, Royal Victoria Hospital, Belfast, Northern Ireland, UK

${ }^{2}$ Edinburgh University, Edinburgh, Scotland, UK

${ }^{3}$ Royal Academy of Engineering Centre of Excellence in Sustainable Building Design, School of Energy, Geosciences, Infrastructure and Society, Heriot-Watt University, Edinburgh, Scotland, UK

${ }^{4}$ Shiley Eye institute, La Jolla, CA, USA

${ }^{5}$ Centre for Clinical Brain Sciences, School of Clinical Sciences, The University of Edinburgh, Edinburgh, Scotland, UK

${ }^{6}$ Ophthalmology, Princess Alexandra Eye Pavilion, Edinburgh, Scotland, UK

Correspondence:

S Sii, Department of Ophthalmology, Royal Victoria Hospital, 274 Grosvenor Rd, Belfast, BT12 6BA, Northern Ireland, UK Tel: +07375128476; Fax: 01315363897. E-mail: samanthasii@ doctors.org.uk

Received: 15 May 2017 Accepted in revised form: 22 October 2017 Published online: 8 December 2017 
in understanding treatment-related psychosocial issues. ${ }^{7}$ Although there have been qualitative studies exploring patients' experience of intravitreal therapy (IVT), which have explored levels of anxiety and discomfort, there remains a lack of information investigating patients' expectations of the IVT given and factors predicting changes in expectations during a course of treatment.7,8

In order to address this need we explored patient's expectations of treatment at a regional AMD centre.

\section{Aims}

The primary aim of this study was to explore changes in patients' expectations during a course of IVT treatment for AMD.

Secondary aims were to explore psychosocial factors during the course of injections including pain, anxiety, preference for doctors or trained nurses to carry out injections, how worthwhile patients rated their visit, and whether they would choose to stop injections.

\section{Materials and methods}

This study was carried out in the AMD unit at the Princess Alexandra Eye Pavilion (PAEP), Edinburgh, that at the time served south-east Scotland. Patients were included in the study if they had been listed for IVT, had been diagnosed with wet AMD, and had completed at least one previous IVT. Additionally, patients needed to be able to have adequate hearing and sight to be able to complete interview questions. Patients were first asked if they could see the questions on paper printed with Arial font 38 and to hear and understand the interviewer. Patients were randomly selected from the clinic list and interviewed independently in a private room by the interviewer. They were verbally guided through each question in a semi-structured interview to reduce bias in understanding. The patients were all treated using a pro re nata (PRN) treatment strategy using a standardised protocol in a treatment room setting by the same healthcare team. At each visit, patients underwent a full ophthalmic examination of both eyes, which included best corrected visual acuity (BCVA) measured using Early Treatment Diabetic Retinopathy Study (ETDRS) letters.
BCVA was taken as latest spectacle wear plus pin hole correction. Refraction was not performed at each visit. Follow-up was initially monthly. A decision to retreat was made on patients if there was evidence of intra- and/or sub-retinal fluid on spectral domain ocular coherence tomography (SD-OCT). Follow-up schedules were decided by the ophthalmologist depending on activity and varied between 1 and 4 months. Patients were able to phone in to a dedicated receptionist or to an answer machine after hours to change appointments and new appointments were provided within 2 weeks if a change of date was required. Consent was sought for every patient in line with the principles laid out by the Helsinki Declaration. Ethics approval was received from the local research ethics committee.

\section{Survey tool}

A seven-item questionnaire addressing different domains was designed by consultant ophthalmologists at Princess Alexandra eye pavilion. The questionnaire was adapted from the previously validated Macular Treatment Satisfaction Questionnaire (MacTSQ). ${ }^{9}$ MacTSQ was developed to provide a means of evaluating satisfaction levels with therapies for macular disease. It consists of response options on a 6-point Likert scale to rate levels of satisfaction. As this study focussed on patients' expectations and other psychosocial domains, the questionnaire was adapted to a visual analogue scale for the purposes of this study (Table 1).

A pilot study was performed to refine the questionnaire in 2 phases of 10 patients each. Each interview lasted between 5 and $10 \mathrm{~min}$. The questionnaire was administered by a semistructured interview technique, whereby the interviewer guided respondents through each question independently.

Demographic information and other details were recorded separately from patient case notes. Other data included were BCVA data of both eyes recorded as better and worse eyes taken from patients' notes. BCVA at first visit and most recently were recorded to represent objective visual acuity. BCVA was taken as latest spectacle wear plus pin hole correction. Refraction was not performed at each visit. Visual acuity was recorded

Table 1 Questionnaire

I When you were first offered the injections, how high were your expectations on its usefulness for your vision? (0-10)

II After having the injections for some time, how high are your expectations on its usefulness for your vision? (0-10)

III How painful/uncomfortable do you feel when given the injections? (0-10)

IV How anxious do you feel when coming for the injections? (0-10)

V How worthwhile do you think is time spent coming for the injections? (0-10)

VI Are you happy for a nurse to carry out the injections? YES/NO

VII Will you choose to stop coming for the injections if your vision continues to deteriorate? YES/NO 
using LogMAR units that utilised the ETDRS chart. ${ }^{10}$ For patients who could only count fingers or perceive hand motion, the equivalence in LogMAR units was recorded. ${ }^{11}$ Additionally, we noted subjective visual acuity, in terms of whether patients perceived their vision to have become worse, maintained, or improved in the context of carrying out their activities of daily living (ADLs). ${ }^{12}$

\section{Statistical analysis}

The primary outcome of this study was ascertaining changes in expectation. Starting expectations and final expectations were measured and any difference categorised into groups 1,2 , and 3 , that is $1=(-5$ to -1$)$ representing rise in expectation; $2=$ (zero) representing no change; and $3=(1$ to 9$)$ representing fall in expectation. Any significant difference between the groups was determined using chi-square test.

The secondary outcome of this study was to ascertain pain, anxiety levels, how worthwhile patients rated their visit, whether they were happy with a nurse practitioner or doctor performing injections, and whether they would choose to stop injections if their vision continue to deteriorate. Using a multinomial regression with latent class analysis, ${ }^{13}$ significant factors that could predict these psychosocial domains were determined.

\section{Results}

A total of 53 patients were approached for the study, 2 declined being interviewed, and 1 could not complete the interview because of inability to comprehend the questions adequately. The characteristics of patients included in the study are summarised in Table 2. Approximately $44 \%$ of patients in this study have other comorbid diagnoses including dry AMD, glaucoma, cataracts, diabetic retinopathy, retinal microaneurysms, and retinal vein occlusion. Fourteen eyes were found to be the second treated eyes.

Table 2 Showing characteristics of patients interviewed for this study

\begin{tabular}{lll}
\hline Characteristics of patients in study, $\mathrm{n}=50$ & & $\mathrm{n}(\%)$ \\
\hline Gender & Men & $19(38)$ \\
& Women & $31(62)$ \\
Age & Range 59-91 & \\
& Mean 80.1 & \\
Duration of AMD since diagnosis & Mean 39.2 & \\
(months) & & \\
Presence of comorbid eye conditions & Yes & $22(44)$ \\
& No & $28(56)$ \\
Total number of injections received & Mean 14.08 & \\
Accompanied & Yes & $32(64)$ \\
& No & $18(36)$ \\
\hline
\end{tabular}

\section{Best corrected visual acuity}

Change in BCVA in this study was categorised into three different categories as per previous studies comparing visual outcome in AMD.9,14 The three categories were worse BCVA (loss of $>15$ ETDRS letters), maintained BCVA (loss of $<15$ ETDRS letters), and improved BCVA (gain of $>15$ ETDRS letters) (Figure 1). The BCVA of $\sim 60 \%$ of patients $(n=27-30)$ was maintained, $30 \%$ $(n=13-17)$ became worse, and only $8.5 \%(n=4)$ showed an improvement. However, subjective vision was perceived to have improved in $31.9 \%(n=15)$ of patients despite the contradictory findings in the objective assessment.

A visual analogue scale from 0 to 10 was created as a tool for measuring five different parameters of the patient experience (Figure 2). Patient's expectations were recorded for starting expectations before the first injection and expectations at the point of interview. Greater than $50 \%$ of patients had starting expectations of $\geqslant 7$, whereas $75 \%$ patients had anxiety and pain levels of $<6$ and $<5$, respectively. Except some outliers, most patients rated their visit as $\geqslant 9$ in terms of whether it was worthwhile. Also, 68\% would choose to continue injections even if their vision continued to deteriorate, whereas $32 \%$ would choose to stop. It should be noted that $78 \%$ of patients were happy for nurses to perform the injections, whereas $22 \%$ preferred a doctor.

\section{Changes in expectation and its predicting factors}

Significantly more patients had either a fall or no change in expectations $\left(\chi^{2}=5.2\right.$, d.f. $=1, P=0.02$.) Significant factors predicting a fall in expectations were higher starting expectations $(P=0.00001)$ as shown in (Figure 3a), objective loss in BCVA in the better eye $(P=0.008)$ as shown in (Figure $3 \mathrm{~b})$, and a reduced subjective change in BCVA $(P=0.013)$ as shown in (Figure 3c). Changes were represented as 1, 2, or 3 representing a rise, no change, or fall in expectations respectively. Differences between the bars were validated using the Mann-Whitney $U$-test, where $P<0.05$ was classed as significant. Being treated for first eye $(n=35)$ or second eye $(n=14)$ made no significant difference to the change in expectations (Mann-Whitney $U$-test, $P=0.604$ ).

\section{Pain and anxiety level}

A statistically significant correlation was found between subjective pain and anxiety $(P<0.01)$ using Pearson's correlation $(r=0.597)$. In addition, there was a statistically significant difference in pain and anxiety level depending on whether patients were accompanied to the injections 


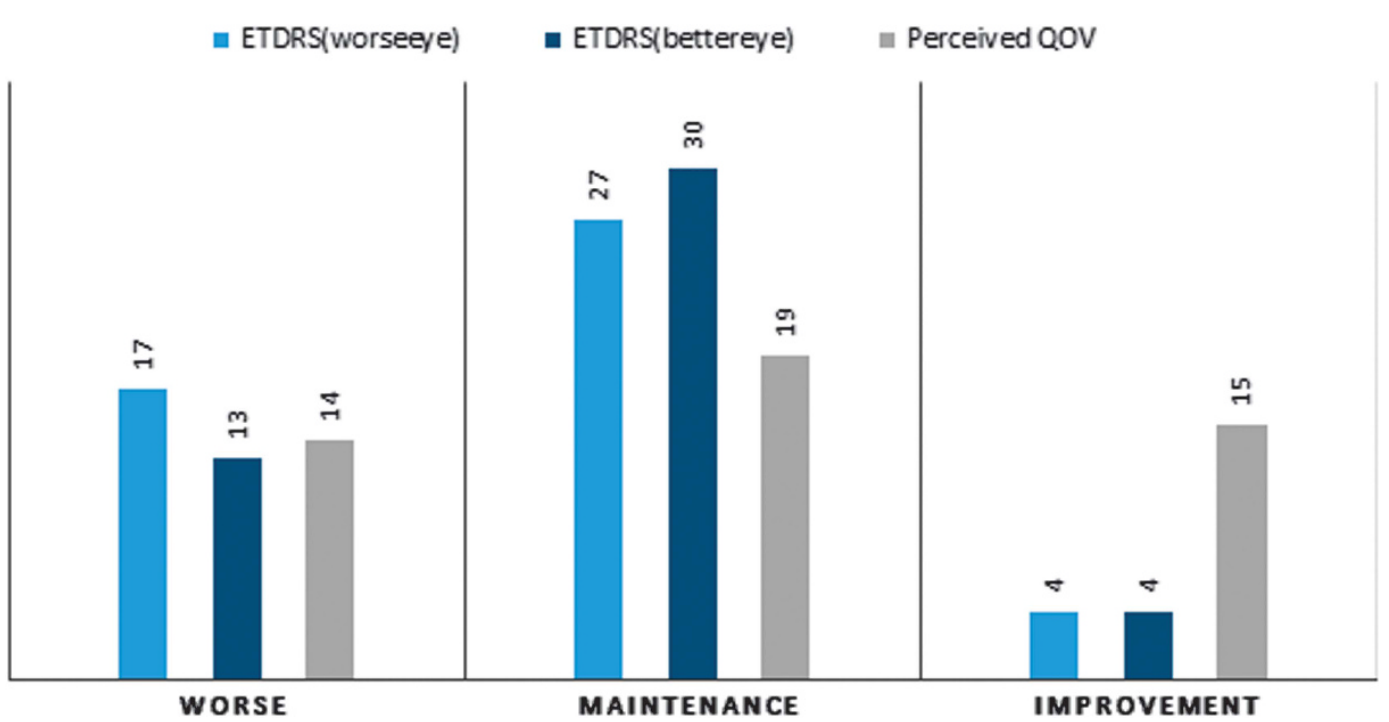

Figure 1 Visual outcome (\%) in (1) ETDRS letters lost or gained in both better and worse eyes and (2) perceived quality of vision.

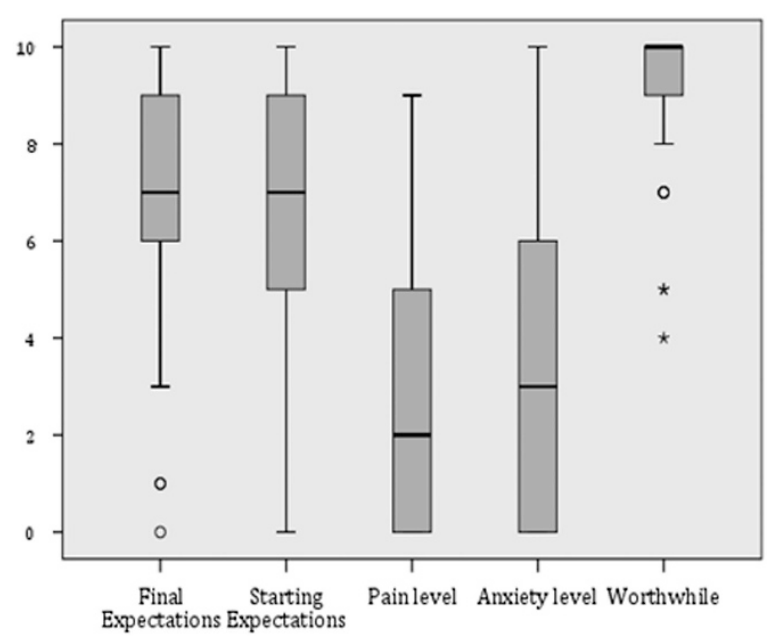

Figure 2 A summary of patient responses to questions on the visual analogue scale using a box and whisker plot.

by a friend or relative (pain $\bar{x}=2.67$, anxiety $\bar{x}=2.50$ ) or if the patients were not accompanied $(P<0.05)$.

\section{Factors predicting a choice to stop injections}

This study reports a statistically significant relationship between mean final visual acuity in the worse eye and a decision to stop the injections if vision continued to deteriorate $(P=0.026,95 \% \mathrm{CI})$. People with a worse final BCVA $(\bar{x}=0.98)$ were more likely to stop attending for the injections compared with people with better final BCVA $(\bar{x}=0.71)$.

\section{Discussion}

This study aims to investigate unmet patient expectations that is an important aspect of every patient's journey in the treatment of AMD. This is distinct from measuring patient satisfaction as this may fluctuate at different time points in their treatment. Hence, to achieve this purpose, a visual analogue scale that depicts change in expectations was utilised instead of using the previously validated Macular Degeneration Treatment Satisfaction Questionnaire that measures patient satisfaction. ${ }^{9}$

In this study, patients reported relatively low levels of pain and anxiety from treatment with intravitreal injections. This is similar to the findings in previous studies. ${ }^{15-17}$ The largest of these studies sampled 225 patients, where $75 \%$ of the respondents reported scores of $<6$ for pain and anxiety. ${ }^{16}$ This could be because of the high average number of injections patients have had before the time when they were interviewed $(\bar{x}$ injections $=14)$. A systematic review of patient experiences during IVT injections found that patients familiarised themselves with the injection experience after the first few injections. ${ }^{15}$ This finding concurs with a study that found that $93 \%$ of patients were neither anxious nor fearful when being treated with IVT. ${ }^{16}$ A qualitative study found that older age groups tended to underreport pain and anxiety. ${ }^{15}$ The mean age of participants reported in those studies were 74 (see Segal et $a l^{17}$ ) and 79 (see Boyle et $a l^{15}$ ) respectively, and most of them had prior injections that was similar finding to our study. However, these studies collectively highlight that a minority of patients do still feel significant pain and anxiety even after repeated injections. Pain was correlated with anxiety levels in a similar manner according to a previous study. ${ }^{17}$ The use of music has previously been shown to ameliorate both anxiety and pain. ${ }^{18}$ Our study found that being accompanied to the clinic could also significantly reduce levels of both pain and anxiety. 

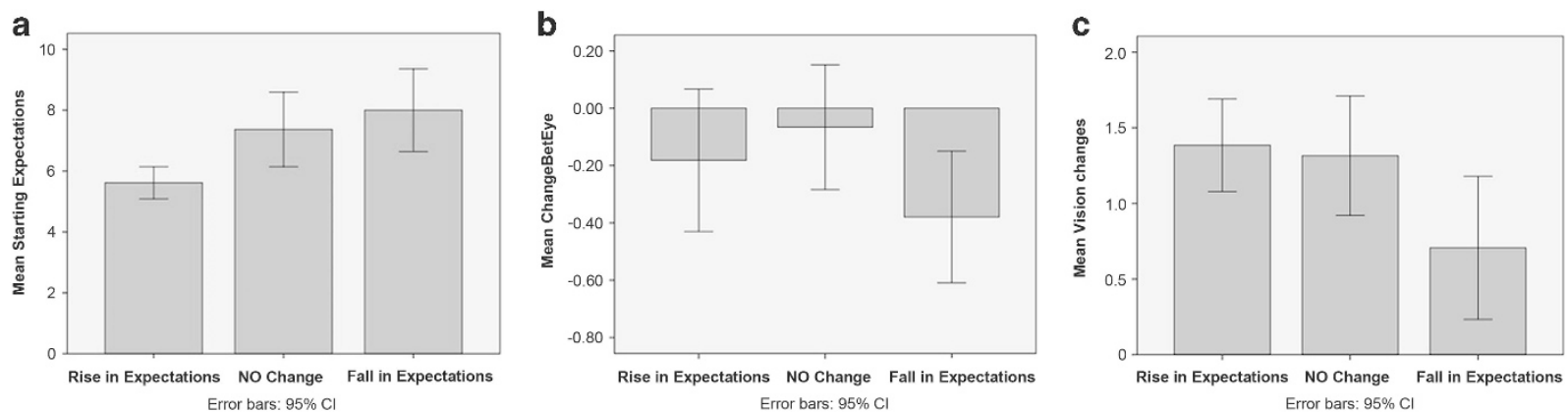

Figure 3 Bar charts summarising changes in patient expectations with (a) mean starting expectation, (b) change in best corrected visual acuity in better eye, and (c) subjective change in visual acuity (error bars $=95 \%$ confidence interval).

This study also investigated patient's expectations about the usefulness of IVT. Our study found that a significantly greater number of patients in the AMD clinic showed a fall in expectation over time than a gain of expectations. This is perhaps unsurprising in the light of findings from other services dealing with reduced vision. AMD patients in a low vision clinic also had unrealistic expectations of what a low vision clinic could provide. ${ }^{8}$ Patients' unrealistic initial expectations may be because of inadequate provision of information during consent.

A patient satisfaction survey showed that $34 \%$ patients received no information about monitoring their macular disease and $22 \%$ received no information about disease progression. 7,8

Unrealistic patient expectations at the start of a course of treatment could also explain why objective or subjective decline in BCVA could result in a fall in of lingering hope, in addition to the sense of trust towards their clinicians. ${ }^{19}$ However, a large minority of patients (32\%) would choose to stop injections at some point if their vision continued to deteriorate. This highlights the need for patients to be allowed the opportunity to discuss stopping treatment especially if disease progression proves to have little or no perceived benefit.

This study was limited by its small sample size. The BCVA outcomes of patients in this study are however similar to two other retrospective case series, Zhu et al ${ }^{20}$ and Jacob et $a l^{21}$ (see Supplementary Information). A second limitation is the use of a fixed structured questionnaire. This limited the capture of psychosocial issues that could be explored compared with an openended format. ${ }^{19}$ However, the visual analogue scale has the benefit of assigning numerical values to different domains, turning them into measurable variables that can be analyzed and compared with other variables.

Structured questioning also reduces interviewer bias by ensuring that the same set of questions were asked even though this does not remove interviewee bias. Future studies on patient expectations using the same scale would be useful in order to explore its application in other clinical setting for other conditions.

In conclusion, pitching starting expectations at the right level by providing appropriate information about disease trajectory is important in order to avoid a fall in expectations along a course of treatment. Most important of all, the service implications of our findings are that patients should be provided with the time and space to permit individualised discussion at specified time points in their treatment plan and this should be emphasised when consenting for multiple IVT procedures. Further work is needed to define the factors that determine expectation levels and the most effective approach to calibrating realistic expectations for patients with AMD. ${ }^{22-25}$

\section{Summary}

\section{What was known before}

- Patients with wet AMD who receive IVT in the long term face psychosocial issues that could hinder compliance, including unmet patient expectations that have not been adequately explored.

- Pain and anxiety during IVT decreases significantly over time, but there is still lack of good-quality studies and methods to ameliorate these factors.

- More AMD departments now have both doctor- and nurse-led clinics but lack of understanding on whether patients have preference for one or the other.

What this study adds

- Fall in patient expectations regarding their treatment is a concern found from this study.

- The only modifiable factor related to fall in patient expectations is high starting expectations as decline in BCVA is unavoidable.

- Patients with worse final BCVA are more likely to stop injections in the future if their vision continues to deteriorate.

- Most patients are contented for nurses to carry out injections instead of doctors.

- Functional vision is an important element to assess among AMD patients and further work should be done to determine factors that could enhance functional vision. 


\section{Conflict of interest}

The authors declare no conflict of interest.

\section{Acknowledgements}

This is an original study that is the collaborative efforts of all the authors in this study, with support from patients and staff members in the PAEP.

\section{References}

1 Owen CG, Jarrar Z, Wormald R, Cook DG, Fletcher AE, Rudnicka AR. The estimated prevalence and incidence of late stage age related macular degeneration in the UK. $\mathrm{Br} J$ Ophthalmol 2012; 96: 752-756.

2 Minassian D, Reidy A, Lightstone A, Desai P. Modelling the prevalence of age-related macular degeneration (2010-2020) in the UK: expected impact of antivascular endothelial growth factor (VEGF) therapy. Br J Ophthalmol 2011; 95: 1433-1436.

3 Shalaby A, Lewis K, Bush K, Meredith P, Simplicio S, Lockwood A. Licence to save: a UK survey of anti-VEGF use for the eye in 2015. Eye 2016; 30: 1404-1406.

4 Keenan TD, Wotton CJ, Goldacre MJ. Trends over time and geographical variation in rates of intravitreal injections in England. Br J Ophthalmol 2012; 96: 413-418.

5 Malik A, Cassels-Brown A, Wormald R, Gray M. Better value eye care for the 21st century: the population approach Br J Ophthalmol 2013; 97(5): 553-557.

6 Basheer K, Mensah E, Khanam T, Minakaran N. Visual outcomes of age-related macular degeneration patients undergoing intravitreal ranibizumab monotherapy in an urban population. Clin Ophthalmol 2015; 9: 959-965.

7 Mitchell J, Bradley P, Anderson S, Ffytche T, Bradley C. Perceived quality of health care in macular disease: a survey of members of the Macular Disease Society. $\mathrm{Br} J$ Ophthalmol 2002; 86(7): 777-781.

8 Crossland M, Gould E, Helman C, Feely M, Rubin G. Expectations and perceived benefits of a hospital-based low vision clinic: results of an exploratory, qualitative research study. Vis Impair Res 2007; 9(2-3): 59-66.

9 Mitchell J, Brose LS, Bradley C. Design of a measure of satisfaction with treatment for macular degeneration (MacTSQ). Qual Life Res 2007 A-120: Abstract 1150.

10 Schmier JK, Hulme-Lowe CK. Cost-effectiveness models in age-related macular degeneration: issues and challenges. Pharmacoeconomics 2015; 34(3): 259-272.

11 Lange C, Feltgen N, Junker B, Schulze-Bonsel K, Bach M. Resolving the clinical acuity categories "hand motion" and 'counting fingers' using the Freiburg Visual Acuity Test (FrACT). Graefes Arch Clin Exp Ophthalmol 2009; 247(1): 137-142.

12 Colenbrander A. Visual Standards - Aspects and Ranges of Vision Loss with Emphasis on Population Surveys. International
Council of Ophthalmology: Sydney, Australia, 2002 Available from http://www.icoph.org/downloads/ visualstandardsreport.pdf. (assessed on 4 April 2016).

13 Magidson J, Vermunt JK. Latent class factor and cluster models, bi-plots and related graphical displays. Sociol Methodol 2001; 31: 223-264.

14 Joussen A, Lehmacher W, Hilgers R, Kirchhof B. Is significant relevant? Validity and patient benefit of randomized controlled clinical trials on age-related macular degeneration. Survey Ophthalmol 2007; 52(3): 266-278.

15 Boyle J, Vukicevic M, Koklanis K, Itsiopoulos C. Experiences of patients undergoing anti-VEGF treatment for neovascular age-related macular degeneration: a systematic review. Psychol Health Med 2015; 20(3): 296-310.

16 Chua P, Mitrut I, Armbrecht A, Vani A, Aslam T, Dhillon B. Evaluating patient discomfort, anxiety, and fear before and after ranibizumab intravitreous injection for wet age-related macular degeneration. Arch Ophthalmol 2009; 127(7): 936-945.

17 Segal O, Segal-Trivitz Y, Nemet A, Cohen P, Geffen N, Mimouni M. Anxiety levels and perceived pain intensity during intravitreal injections. Acta Ophthalmol 2015; 94(2): 203-204.

18 Chen X, Seth R, Rao V, Huang J., Adelman R. Effects of music therapy on intravitreal injections: a randomized clinical trial. J Ocul Pharmacol Ther 2012; 28(4): 414-416.

19 McCloud C, Lake S. Understanding the patient's lived experience of neovascular age-related macular degeneration: a qualitative study. Eye 2015; 29: 1561-1569.

20 Zhu M, Chew JK, Broadhead GK, Luo K, Joachim M, Hong T et al. Intravitreal ranibizumab for neovascular Age-related macular degeneration in clinical practice: five-year treatment outcomes. Graefes Arch Clin Exp Ophthalmol 2015; 253(8): 1217-1225.

21 Jacob J, Brié H, Leys A, Levecq L, Mergaerts F, Denhaerynck K. Six-year outcomes in neovascular age-related macular degeneration with ranibizumab. Int J Ophthalmol 2017; 10(1): 81-90.

22 Tano Y, Wolf S. Ranibizumab (Lucentis) in neovascular age-related macular degeneration: evidence from clinical trials. Br I Ophthalmol 2010; 94(1): 2-13.

23 Basheer K, Mensah E, Khanam T, Minakaran N. Visual outcomes of AMD patients undergoing intravitreal ranibizumab monotherapy in urban populations. Clin Ophthalmol 2015; 9: 959-965.

24 Wong TY, Chakravarthy U, Klein R, Mitchell P, Zlateva G, Buggage $\mathrm{R}$ et al. The natural history and prognosis of neovascular age-related macular degeneration: a systematic review of the literature and meta-analysis. Ophthalmology 2008; 115(1): 116-126.

25 Rosenfeld PJ, Shapiro H, Tuomi L, Webster M, Elledge J, Blodi B. Characteristics of patients losing vision after 2 years of monthly dosing in the phase III ranibizumab clinical trials. Ophthalmology 2011; 118(3): 523-530. 